\title{
Speckle-Visibility Spectroscopy of Depolarized Dynamic Light Scattering
}

\author{
David Bossert, ${ }^{\dagger}$ Jens Natterodt, ${ }^{\dagger}$ Dominic A. Urban, ${ }^{\dagger}$ Christoph Weder, ${ }^{\dagger}$ C Alke Petri-Fink, ${ }^{\dagger, \ddagger \subset}$ \\ and Sandor Balog* ${ }^{\dagger}+(1)$ \\ ${ }^{\dagger}$ Adolphe Merkle Institute, University of Fribourg, Chemin des Verdiers 4, 1700 Fribourg, Switzerland \\ ${ }^{\ddagger}$ Chemistry Department, University of Fribourg, Chemin du Musée 9, 1700 Fribourg, Switzerland
}

Supporting Information

\begin{abstract}
We show that the statistical analysis of photon counts in depolarized dynamic light scattering experiments allows for the accurate characterization of the rotational Brownian dynamics of particles. Unlike photon correlation spectroscopy, the technique is accurate even at low temporal resolution and enables discontinuous data acquisition, which offers several advantages. To demonstrate the usefulness of the method, we present a case study in which we analyze aqueous suspensions of tunicate cellulose nanocrystals and silica particles, and discuss aspects that are specific to particle sizing.
\end{abstract}

\section{INTRODUCTION}

Micro- and nanoparticles are found in both academic and industrial laboratories in many different forms, including powders, dispersions, colloidal suspensions, and others. ${ }^{1}$ They are used as the basis for composite materials, paints and inks, mining solutions, soaps and detergents, abrasives and polishes, catalysts, cements, energy devices, food products, electronics, textiles, biomedicine, pharmaceutical diagnostics, and therapeutics. Given that many physical, physicochemical, and biological phenomena are influenced by particle size (distribution) and interparticle interactions, exploring their fundamental aspects is of paramount importance, and it is without a doubt that advances in understanding the fundamental properties of (nano)particles have in many instances been driven by the development of the experimental techniques available.

Scattering techniques, which probe the characteristic features and principles of structure and dynamics at the nanometer scale, have been at the forefront of soft matter research. ${ }^{2}$ Arguably, the most often used technique is dynamic light scattering (DLS), also known as photon correlation spectroscopy (PCS) or quasielastic light scattering. The popularity of this technique is likely rooted in the fact that a wide range of time- and length-scales can be covered and that the procedure of data collection itself is straightforward. In an experiment, a collimated coherent laser beam illuminates an ensemble of particles that are subjected to Brownian motion, and the interference of the scattered waves forms a grainy random pattern. This is the so-called speckle pattern, and the grains of this pattern, which are spatially coherent domains, are called speckles. The constant movement of the particles results in a continuously evolving interference pattern, which is described

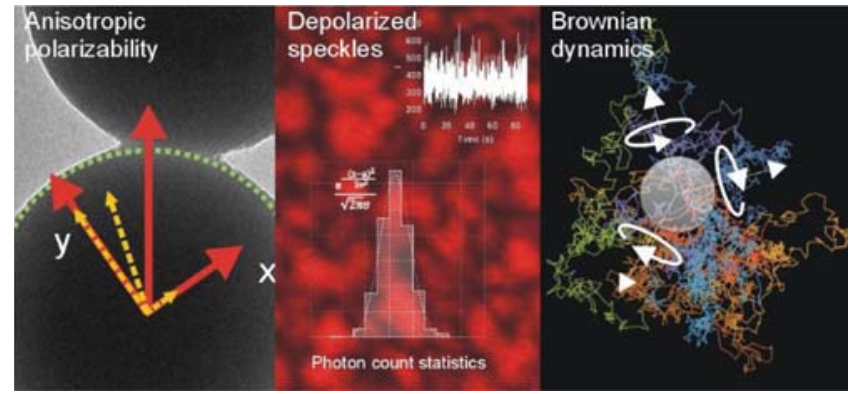

by the appearance and disappearance of optical maxima and vortices throughout the pattern, and appears as a chaotic fluctuation in the brightness of a given speckle. The statistical properties of these random temporal fluctuations carry quantitative information about the Brownian dynamics. 3 ,4

The optical anisotropy of particles adds another level of complexity to DLS, which can be exploited in depolarized dynamic light scattering (also referred to as dynamic depolarized light scattering, DDLS). ${ }^{5}$ Depolarization is a direct consequence of anisotropic polarizability. In the case of elastic scattering, the induced dipole moment of an anisotropic particle-whose polarizability is dependent on its orientation with respect to the laser's polarization-will contain a component that is perpendicular to the laser's polarization. The particle's orientation uniquely determines the amplitude of this perpendicular component, and if the particle turns, the amplitude will change accordingly. Thus, upon rotational diffusion, the amplitude of depolarized scattering will fluctuate, following the particle's orientation and optical anisotropy. As a result, DDLS can provide direct access to probing rotational Brownian dynamics. This provides opportunities that DLS does not offer, and DDLS has been used for characterizing a wide range of particle systems (Supporting Information, DDLS and particle system). We have also shown recently that DDLS can be employed to accurately characterize the number-averaged size distribution of nanoparticles, ${ }^{6}$ and it has been demonstrated recently by others that the concept behind DDLS can 
be successfully adapted to analyze partially depolarized scattering. $^{7-9}$

Although PCS has been used for 50 years and is considered to be a mature technique, certain limitations persist. ${ }^{10}$ Highstandard instruments are delicate and costly; the photon detector unit must be able to perform at a high speed, with sampling times within the range of $0.1-1 \mathrm{~ns}$, with a suitably low signal-to-noise ratio, and without signal-processing artefacts. The choice of the correlator unit is crucial. Although many commercial digital correlators are available, most operate in ways that are different and far from the ideal mathematical concept, ${ }^{11,12}$ and noise and dead time must be carefully considered. ${ }^{13}$ Multiangle studies either require a precision goniometer or multiple detectors and correlators. ${ }^{14}$ Given that, in the case of DDLS, the correlation can show a decay that is several orders of magnitude faster than that in DLS, these requirements become even more demanding. The necessary high temporal resolution is not without consequences, and to mitigate the impact of shot noise and detector after-pulsing, the scattering signal must be split and cross-correlated using two independent detector and correlator units.

Here, we propose an alternative that can eliminate these problems. We demonstrate that the analysis of the statistical moments of the photon count distribution is suitable for characterizing rotational and translational Brownian dynamics, although the smallest temporal resolution $(\sim 10 \mathrm{~Hz})$ of the experiment is 8 orders of magnitude smaller than the one typically used in PCS $(\sim 1 \mathrm{GHz})$. To the best of our knowledge, this approach is new to DDLS. We present case studies addressing the particle size analysis of dilute aqueous suspensions of (1) tunicate cellulose nanocrystals (tCNCs) isolated from club sea squirts (Styela clava) and (2) silica particles synthesized from tetraethyl orthosilicate (TEOS), $\mathrm{SiO}_{2}-\mathrm{A},-\mathrm{B},-\mathrm{C}$, and $-\mathrm{D}$, with a nominal diameter of around 100 , 200, 300, and $400 \mathrm{~nm}$, respectively. While tCNCs exhibit a highly anisotropic shape and are dominantly crystalline, the $\mathrm{SiO}_{2}$ particles are almost spherical and amorphous. Small deviations from the spherical shape and the internal heterogeneities of the mass density, however, result in anisotropic polarizability and lead to appreciable depolarized light scattering at low scattering angles.

\section{MATERIALS AND METHODS}

tCNCs. tCNCs were isolated from Styela clava, following a procedure described in detail elsewhere. ${ }^{15}$ After hydrolysis, the isolated tCNCs were sonicated for $8 \mathrm{~h}$ and kept suspended at a concentration of $\sim 1 \mathrm{mg} / \mathrm{mL}$ in deionized water. For light scattering studies, the suspension was diluted until multiple scattering was found to be negligible.

Silica Particles. The $\mathrm{SiO}_{2}$ particles were synthesized following a procedure based on the Stöber synthesis. ${ }^{16}$ To obtain the $\mathrm{SiO}_{2}$-A particles, a mixture of ethanol $(162 \mathrm{~mL})$, water (18 $\mathrm{mL}$, MilliQ grade), and ammonium hydroxide (7.8 $\mathrm{mL}$ ) was transferred to a flask, and heated to $70{ }^{\circ} \mathrm{C}$ with the help of an oil bath, the mixture was stirred with a magnetic stirrer and equilibrated for $1 \mathrm{~h}$ before TEOS $(22 \mathrm{~mL})$ was added. The mixture was then stirred for several hours. Afterward, the ethanol was removed from the suspension by evaporation under a reduced atmosphere. Following the removal of the ethanol, the suspension was transferred to a dialysis tube, and submersed in a large beaker filled with water. To obtain the $\mathrm{SiO}_{2}-\mathrm{B}$ particles, a mixture of ethanol $(161 \mathrm{~mL})$, water $(13.5 \mathrm{~mL}$, MilliQ grade), and ammonium hydroxide (7.8
$\mathrm{mL}$ ) was transferred to a flask, and heated to $50.6{ }^{\circ} \mathrm{C}$ with the help of an oil bath, while being stirred with a magnetic stirrer. Next, TEOS $(22.2 \mathrm{~mL})$ was transferred rapidly to the round bottom flask, and the mixture was stirred for several hours. Afterward, the ethanol was removed from the suspension by evaporation under a reduced atmosphere. Following the removal of the ethanol, the suspension was transferred to a dialysis tube, and submersed in a large beaker filled with water. The water was continuously stirred and refreshed over the course of a few days. To obtain the $\mathrm{SiO}_{2}-\mathrm{C}$ and $-\mathrm{D}$ particles, a mixture of water $(13.5 \mathrm{~mL})$, ammonium hydroxide $(40.9 \mathrm{~mL})$, and $\mathrm{EtOH}(174 \mathrm{~mL})$ was heated to $60^{\circ} \mathrm{C}$ and equilibrated for $1 \mathrm{~h}$, before TEOS $\left(21 \mathrm{~mL}, \mathrm{SiO}_{2}-\mathrm{B}\right.$ and $\left.23 \mathrm{~mL}, \mathrm{SiO}_{2}-\mathrm{C}\right)$ was added. The mixture was stirred overnight, and then the suspension was left to cool to room temperature. The suspensions were washed and centrifuged at $5000 \mathrm{~g}$ for 20 min and were finally redispersed in water. The ammonium hydroxide $(25 \%$ as solution, Honeywell), ethanol (HPLCgrade, Honeywell), and TEOS (99\%, Merck) were used as received.

Light Scattering. Given that the suspensions might contain some agglomerates and bundles, the suspensions (silica and tCNCs) were left to stand for over $48 \mathrm{~h}$ in a vial, and when collecting light scattering data, a sample was taken from the top of the vial. To ensure that in our experiments multiple scattering did not contribute either to depolarization or to the dynamics of speckle fluctuations, multiple scattering was minimized by using sufficiently dilute suspensions of the particles, and thus each sample was prepared at a low concentration where multiple scattering was negligible. To confirm that contributions from multiple scattering was negligible or at most not significant, we performed conventional DLS (non-depolarized) measurements using a three dimensional (3D)-cross correlation scheme (Supporting Information, multiple scattering). ${ }^{17}$ Data were collected at constant temperature $\left(21^{\circ} \mathrm{C}\right)$ using a commercial goniometer instrument (3D LS Spectrometer; LS Instruments AG, Switzerland). The primary beam was formed by a linearly polarized and collimated laser beam (Cobolt 05-01 diode pumped solid state laser, $\lambda=660 \mathrm{~nm}, P_{\max }=500 \mathrm{~mW}$ ), and the scattered light was collected by single-mode optical fibers equipped with integrated collimation optics. With respect to the primary beam, depolarized scattering was observed via cross-polarizers. The incoming laser beam passed through a Glan-Thompson polarizer with an extinction ratio of $10^{-6}$, and another GlanThompson polarizer, with an extinction ratio of $10^{-8}$, was mounted in front of the collection optics. For the $\mathrm{SiO}_{2}$ particles, data were collected at a scattering angle of $\theta=30^{\circ}$, and for the tCNCs, data were collected at scattering angles of $15^{\circ} \leq \theta \leq 150^{\circ}$.

Photon Correlation. To construct the intensity autocorrelation function, $g_{2}(t)$, the collected light was coupled into two APD detectors via laser-line filters (Perkin Elmer, single photon counting module), and their outputs were fed into a twochannel multiple-tau correlator. To improve the signal-to-noise ratio and to eliminate the impact of detector after-pulsing on $g_{2}(t)$ at early lag times below $1 \mu \mathrm{s}$, these two channels were cross-correlated.

Speckle Visibility and Photon Counting. Without any modifications made, the photon counts of one of the APD detectors were obtained through the same detection line as above, at a sampling rate of $\sim 9.5 \mathrm{~Hz}(0.105 \mathrm{~s}$ integration time). 
Transmission Electron Microscopy. Micrographs were taken with a Tecnai Spirit electron microscope (FEI), operating at $120 \mathrm{kV}$. All images were recorded at a resolution of $2048 \times$ 2048 pixels (Veleta CCD camera, Olympus). Prior to imaging, the $\mathrm{SiO}_{2}$ particles were diluted with ethanol (99.98\%, VWR chemicals) and $5 \mu \mathrm{L}$ of this mixture was drop cast onto a carbon-film square mesh copper grid (CF-300-Cu; Electron Microscopy Sciences) and left to dry under ambient air. For particle characterization, the images were bi-leveled in Image J (National Institutes of Health, NIH) using the default threshold method (IsoData-based variation). ${ }^{18}$ The binary images were analyzed by a built-in routine (Analyze Particles) without separation methods and constraints. Prior to imaging, the suspension of tCNCs was diluted to $0.01 \mathrm{mg} / \mathrm{mL}$, sonicated for $30 \mathrm{~min}$, and one drop of the dispersions was evaporated on a carbon-coated TEM grid in a ventilated oven at $60^{\circ} \mathrm{C}$ for $1 \mathrm{~h}$.

\section{THEORY}

Given that the energy of light is quantized into discrete portions, detecting the intensity of scattered light is never instantaneous, but involves integration over a finite time interval $\tau>0$. Furthermore, detecting a photon is intrinsically random, and the consequence of this is that the number of photons detected during a fixed length of $\tau$ is a random variable. Even if the scattered light does not fluctuate at all and its intensity is constant, the photon count distribution follows a Poisson distribution. This is due to the uncertainty associated with the measurement and inherent to the quantized nature of light. ${ }^{19}$ This phenomenon is referred to as shot noise.

In the presence of Brownian motion and the consequent speckle fluctuations, the photon count distribution becomes broader than a Poisson distribution. It can be shown that the coefficient of variation $(\delta)$ of the photon count distributiondefined as the standard deviation normalized by the mean-is the sum of two independent terms $s^{3,19}$

$$
\delta(\tau)=\frac{\sqrt{\left\langle n(\tau)^{2}\right\rangle-\langle n(\tau)\rangle^{2}}}{\langle n(\tau)\rangle}=\sqrt{\frac{1}{\langle n(\tau)\rangle}+\frac{1}{M(\tau)}}
$$

The coefficient of variation is a dimensionless number that quantifies the degree of variability relative to the mean. The first term of the right side-where $\langle n(\tau)\rangle$ is the average number of photons counted during a series but otherwise fixed sampling interval $\tau$-corresponds to shot noise. The second term-the so-called interference term-corresponds uniquely to the random fluctuation of the speckles. It is well-known that the rate of fluctuation of the depolarized speckles carries information about rotational Brownian dynamics, and hence it can be used to describe the dimensions of the particles suspended. ${ }^{5}$ The rate of the speckle fluctuations is usually quantified by PCS and via the intensity autocorrelation function $g_{2}(t)$ and its relaxation rate. The relaxation rate is inversely proportional to the coherence time $T_{c}$ (also frequently referred to as the correlation time) of the speckle fluctuation. $T_{c}$ represents the length of the period over which the scattered light may be considered to be coherent. It can be shown that when $\tau \gg T_{\mathcal{c}}$ that is, the integration time is much larger than the coherence time of the speckle fluctuation, the interference term in eq 1 is given by ${ }^{20}$

$$
M(\tau) \cong \tau / T_{\mathrm{c}}
$$

The inaccuracy of eq 2 is smaller than $5 \%$ when $\tau / T_{c}>10$, smaller than $1 \%$ when $\tau / T_{c}>50$, and smaller than $0.5 \%$ when $\tau / T_{\mathrm{c}}>100$. Thus, $M(\tau) \cong \tau / T_{\mathrm{c}}$ is in fact a measure of the average number of coherent time intervals included within $\tau$. When $\tau \gg T_{\mathcal{c}}$ the shot noise is negligible because $\langle n(\tau)\rangle \gg$ $M(\tau)$, and the corresponding photon count distribution essentially follows a Gaussian distribution ${ }^{20}$

$$
P(n, \tau) \cong \frac{1}{\sqrt{2 \pi} \sigma} \mathrm{e}^{-(n-\mu)^{2} / 2 \sigma^{2}}
$$

where $\mu=\langle n(\tau)\rangle$ and $\sigma^{2}=\langle n(\tau)\rangle^{2} \cdot M(\tau)^{-1}$. Consequently

$$
M(\tau) \cong \frac{\langle n(\tau)\rangle^{2}}{\sigma^{2}}
$$

To summarize, the coefficient of variation $(\delta)$ can be considered as a measure of the relative visibility of individual speckles, hence the name "speckle-visibility". The visibility of a speckle is proportional to the intensity contrast between bright and dark speckles. Given that the speckle contrast observed is inversely proportional to the integration time, the Brownian dynamics can be characterized by analyzing the photon count distribution of the scattered light.

There are some criteria for this analysis. The coherence time $T_{\mathrm{c}}$ of the speckle fluctuation-defined by Brownian dynamics and the angle of scattering-partially stipulates the experimental parameters required for a successful statistical analysis. Generally, the overall length of the photon trace $(t)$ should be considerably longer than the integration time $(\tau)$, and the sampling time should be considerably longer than the coherence time: $t \gg \tau \gg T_{\mathrm{c}}$. Considering the latter, if $\tau>$ $50 T_{\mathcal{c}}$ eq 2 is accurate within $1 \%$, and therefore this criterion is easily fulfilled. The other condition ensures that the size of the sample is sufficiently large and statistically representative. We define a sample as a finite set of photon counts collected within the integration time $\tau$. A sample has $s=t / \tau$ elements, where $s$ is the sample size. The coefficient of variation is always estimated from a finite sample, which is, in our case, a stream of photon counts consisting of $s>2$ elements.

When considering what should be the overall size of a sample, one must keep in mind that probabilities only give the odds of (complementary) events. Accordingly, one ought to seek a compromise between the expected ranges of accuracy and uncertainty and the amount of experimental evidence collected. The accuracy and precision of determining the coherence time of the speckle fluctuation are ultimately dictated by those of determining the coefficient of variation of the photon counts. The term "accuracy" refers to the closeness of an estimate to the true value (or population value), and the term "precision" refers to the degree of agreement in a series of estimates (Figure SI1).

To determine the influence of the sample size on our statistical analysis, we derived the probability density function (PDF) of the coefficient of variation as a function of sample size $s\left(\delta_{s}\right)$, which is also known as the sampling distribution of the coefficient of variation. We began with McKay's approximation. $^{21,22}$ This approximation addresses finite-size samples drawn randomly from a normally distributed population, and defines a variable $K_{s}$ as the following function of the sample statistic

$$
K_{s}\left(\delta, s ; \delta_{s}\right)=\left(1+\frac{1}{\delta^{2}}\right) \frac{(s-1) \delta_{s}^{2}}{1+\frac{(s-1)}{s} \delta_{s}^{2}}
$$


(a)

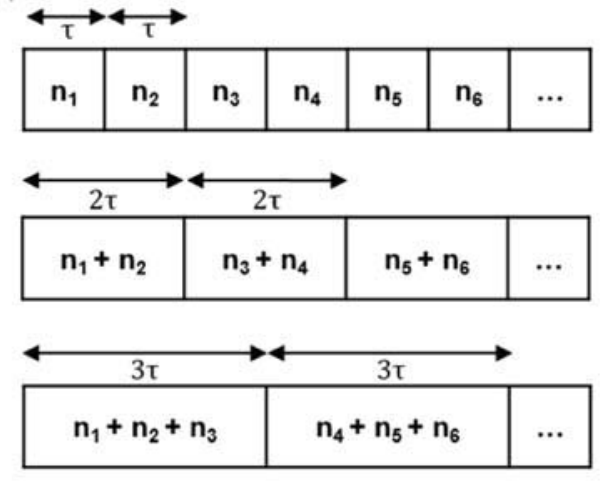

(b)

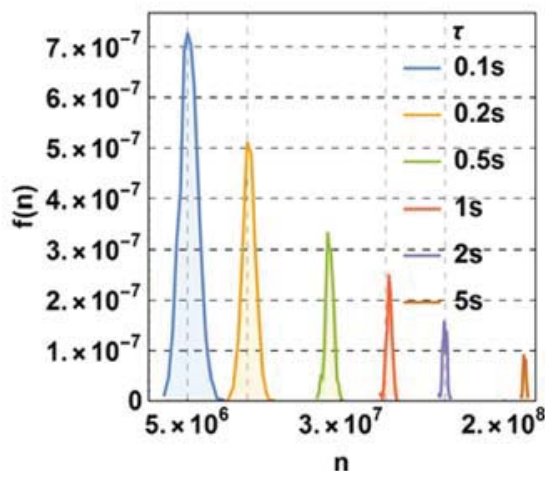

Figure 1. (a) Schematic representation of the simple algorithm used for obtaining photon counts at different integration times from a single trace sampled with $\tau$ time. When $\tau \gg T_{\mathcal{c}}$ any $n_{i}$ and $n_{j}$ are uncorrelated and independent of one another. Using this algorithm, the sample size decreases systematically as the integration time increases. (b) The probability density distribution of photon counts constructed at six integration times (loglin scale). $f(n) \times \mathrm{d} n$ quantifies the probability that the photon count number is found in $\mathrm{d} n$ interval about $n$. The depolarized scattering from a dilute aqueous suspension of tCNCs was observed at $\theta=150^{\circ}$.

where $\delta$ and $\delta_{s}$ are the population and sample coefficients of variation, respectively, and $s$ is the sample size. McKay showed that when $\delta<1 / 3$, the PDF of $K_{s}$ can be described by a central $\chi^{2}$-distribution with $s-1$ degrees of freedom

$$
f_{s-1}\left(K_{s}\right)=\left\{\begin{array}{l}
\frac{2^{(1-s) / 2} \mathrm{e}^{-K_{s} / 2} \cdot K_{s}^{(s-3) / 2}}{\int_{0}^{\infty} K_{s}^{(s-3) / 2} \mathrm{e}^{-K_{s}} \mathrm{~d} K_{s}} K_{s}>0 \\
0
\end{array}\right.
$$

The value limiting $\delta$ ensures that the occurrence of negative numbers has practically zero chance, that is

$$
\int_{0}^{\infty} \frac{1}{\sqrt{2 \pi} \sigma} \mathrm{e}^{-(n-\mu)^{2} / 2 \sigma^{2}} \cong \int_{-\infty}^{\infty} \frac{1}{\sqrt{2 \pi} \sigma} \mathrm{e}^{-(n-\mu)^{2} / 2 \sigma^{2}}=1
$$

The exclusion of negative numbers is fully consistent with photon counting. To calculate the PDF of the sample coefficient of variation $p\left(\delta_{s}\right)$, we transform McKay's approximation $^{23}$

$$
p\left(\delta_{s}\right)=f_{s-1}\left(K_{s}\left(\delta_{s}\right)\right) \times\left|\frac{\partial K_{s}}{\partial \delta_{s}}\right|
$$

After evaluating eq 8 , we obtained the sampling distribution of the coefficient of variation of Gaussian variables

$$
\begin{aligned}
& p\left(\delta, s ; \delta_{s}\right)= \\
& \left(2^{3 / 2-s / 2} \cdot \sqrt{\frac{\left(\delta^{2}+1\right)^{2}(s-1)^{2} s^{4} \delta_{s}^{2}}{\delta^{4}\left((s-1) \delta_{s}^{2}+s\right)^{4}}} \cdot\left(\frac{\left(\frac{1}{\delta^{2}}+1\right)(s-1) s \delta_{s}^{2}}{(s-1) \delta_{s}^{2}+s}\right)^{(s-3) / 2}\right. \\
& \left.\mathrm{e}^{\left(\frac{1}{\delta^{2}}+1\right)(s-1) \delta \delta_{s}^{2} / 2\left((s-1) \delta_{s}^{2}+s\right)}\right) /\left(\int_{0}^{\infty} x^{(s-3) / 2} \mathrm{e}^{-x} \mathrm{~d} x\right)
\end{aligned}
$$

where $\delta$ is the true value (or population parameter), and $s$ is the sample size. We tested the validity of eq 9 against independent computational experiments using pseudorandom numbers. In the Supporting Information, we provide a detailed analysis of the coefficient of variation estimated from a finite set of samples (accuracy and precision of the coefficient of variation). We found excellent agreement between the theory (eq 9) and the results of the computational experiments (Figures SI2 and SI3), and therefore we were confident in applying it further to estimate the expectable accuracy and precision as a function of the sample size. In general, one can collect a sufficient amount of experimental evidence in two ways. One can collect numerous samples of identical size (e.g., $s=25,10$ times) and use their mean value. It can be shown that (eqs $1-6$ of SI) the expected sample mean approaches the true value fast (Figure SI4a), and the expected range of accuracy is already within $3 \%$ when the samples have only 10 elements (Figure SI4c). At this value, the uncertainty is, however, relatively high (Figure SI4b), because the distribution of the coefficient of variation is still broad (Figure SI3). To arrive within 1\% accuracy, on average a sample of $s>26$ elements is sufficient, and the precision is $14 \%$ at this value.

We also considered the particular case when one single sample is collected, and we were interested in the expectable accuracy and precision of the results as a function of its size. As anticipated, when the sample size increases, the probability of accuracy increases (Figure SI5a). For instance, the probability is over 0.9 for finding accuracy within $10 \%$ when the sample size is larger than 150 . To arrive at a probability of 0.9 inside the $1 \%$ interval, the sample size must be 100 times larger. To achieve a probability of 0.99 inside the $1 \%$ interval, the sample size must have more than 36000 elements. Considering photon counting experiments, if $\tau=1 \mu \mathrm{s}$, this bias and sample size would require a less than $0.4 \mathrm{~s}$ long photon trace, which is short indeed. We addressed this the other way around as well, that is, we determined the range of accuracy in which $\delta_{s}$ is expected to fall within a given probability as a function of the sample size. Figure SI5b shows that the accuracy increases as the sample size increases. For example, when the sample size is larger than 147, $\delta_{s}$ is close to $\delta$ within $10 \%$ with a 0.9 probability, whereas a 0.99 probability requires a sample size that is larger than 400 .

We note that eq 9 provides the starting point if one was to quantify the propagation of uncertainty that originates from the statistical fluctuations in determining any function of $\delta_{s}$. If, for example, one is interested in the accuracy and precision of determining $T_{\mathfrak{c}}$ beyond the commonly used small-error approximation, the general case-the PDF of $T_{c}$ as a function of sample size-can be easily obtained by using $s=t / \tau$ and $\delta=$ $T_{\mathrm{c}} / \tau$, and by applying again the rule of transforming random 
(a)

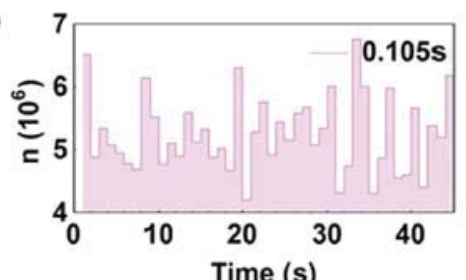

(b)

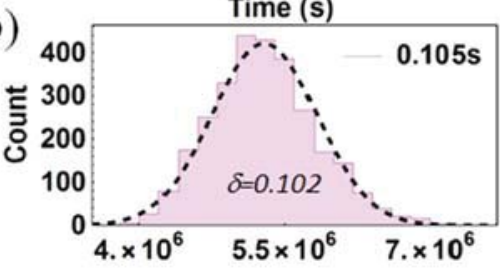

(c)

(d)
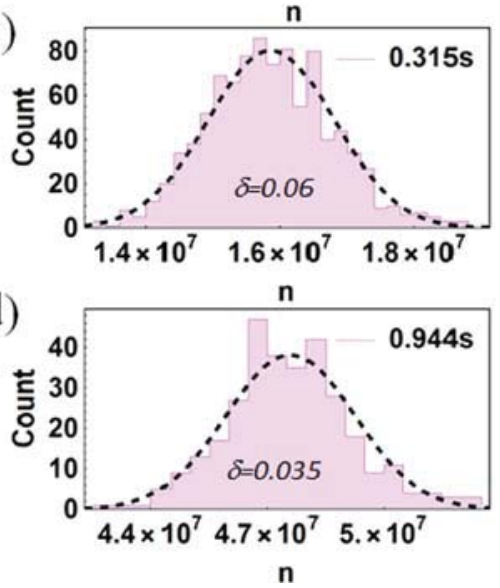

(e)

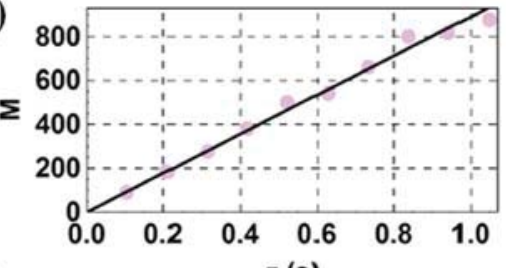

(f)

$\tau$ (s)

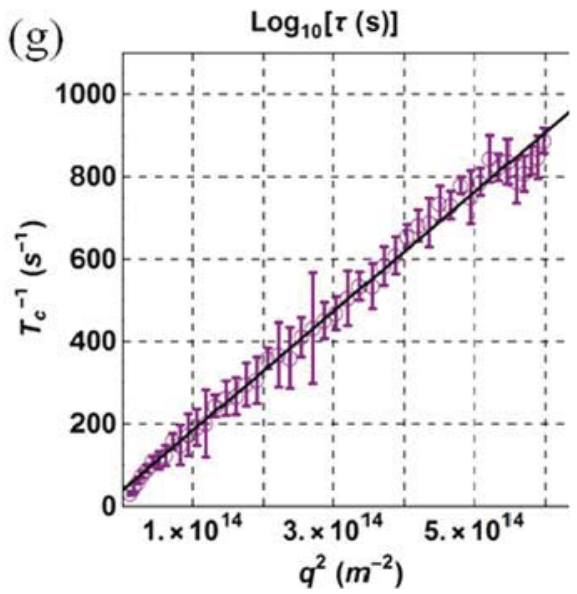

Figure 2. Analysis of photon counts of depolarized scattering from an aqueous suspension of tCNCs. (a-f) the scattering angle was $\theta=150^{\circ}$. (a) Plot showing the numbers of photons counted over $45 \mathrm{~s}$ with $\tau=0.105 \mathrm{~s}$ integration time. (b-d) Distributions of photon counts obtained at three different integration times. The dashed lines show unadjusted Gaussian functions corresponding to the mean and the variance of the respective photon counts. The numbers typed in italic are the coefficients of variation of the photon counts (eq 1). (e) Plot of $M$ as a function of $\tau$ (symbol) and the linear regression to the data pairs. (f) Plot of the logarithms of $M$ and $\tau$ on an extended range $(0.105 \mathrm{~s} \leq \tau \leq 100.5 \mathrm{~s})$. The solid line is redrawn from panel (e), and the dashed line is the extrapolation of the solid line. $(\mathrm{g})$ Plot showing the $q^{2}$-dependence of the relaxation rate determined from depolarized speckle-visibility analysis $\left(15^{\circ} \leq \theta \leq 150^{\circ}\right)$. Each value of $M$ was obtained as shown in panel (e), and $T_{c}$ was obtained using eq 2 . The uncertainty (error bar) is given by the standard deviation determined from 10 measurements per angle. The solid line is a linear regression.

variables. By having the sampling distribution at hand, the expectable accuracy and precision of $T_{c}$ can be calculated in the same manner as that for $\delta$ and for any sample size. Naturally, the same is true for $T_{\mathrm{c}}^{-1}$ and the hydrodynamic radius.

\section{RESULTS}

We collected photons using a commercial instrument designed and built for correlation spectroscopy. The low concentration and the nonfocused beam used in our experiments ensured that the speckle fluctuations were independent of one another. ${ }^{24}$ There was only one available time resolution to count photons. The sampling rate was relatively low, $\sim 9.5 \mathrm{~Hz}$, which corresponds to an integration time of $\tau=0.105 \mathrm{~s}$. Accordingly, we had to collect photons during relatively long measurements. Photons from tCNCs were collected for $t=300 \mathrm{~s}$ and those from $\mathrm{SiO}_{2}$, for $t=600 \mathrm{~s}$. These lengths defined maximum sample sizes of 2857 and 5714, respectively. Photon traces with $2 \tau, 3 \tau, 4 \tau$, and so forth were constructed as illustrated in Figure $1 \mathrm{a}$. Figure $1 \mathrm{~b}$ shows the influence of the integration time on the probability density distribution of photon counts. The mean value is proportional to the integration time, whereas the relative width of the distribution decreases with $\tau$.
tCNCs. Figure 2a shows a part of a photon trace of depolarized light scattering from a dilute aqueous suspension of tCNCs. Indeed, as is predicted by eqs $1-4$, the distributions of photon counts followed normal distributions, and the coefficient of variation $\delta$ decreased with integration time (Figure $2 \mathrm{~b}-\mathrm{d}$ ).

Following eq 4, the dynamics of Brownian motion can be determined via the dependence of $\delta$ on $\tau$. Figure 2e,f shows that $M$ was linearly proportional to $\tau$. Via eq 2 and a linear regression, we determined a coherence time of $T_{c}=1.04 \mathrm{~ms}$. Figure $2 \mathrm{f}$ shows $M$ as a function $\tau$ computed over 3 orders of magnitude. We found that the linear relationship was valid over a wide range, and the agreement between theoretical prediction and experimental evidence was preserved up to $\tau \approx 20 \mathrm{~s}$ $\left(\log _{10}[\tau(s)]=1.3\right)$. Above this value, however, some deviation was appreciable. Given that the sample size was systematically decreasing with increasing $\tau$, we attribute this deviation to statistical fluctuations.

To probe the scattering-angle dependence of the observed Brownian dynamics, we collected photon traces at several scattering angles (Figure $2 \mathrm{~g}$ ). The relaxation rate corresponding to translation diffusion was found to be proportional to $q^{2}$, whereas the relaxation rate of the speckle fluctuations 
corresponding to rotational dynamics was expected to be independent of the scattering angle

$$
T_{\mathrm{c}}^{-1}=6 D_{\mathrm{R}}+q^{2} D_{\mathrm{T}}
$$

The diffusion coefficients, $D_{\mathrm{R}}$ and $D_{\mathrm{T}}$, correspond to rotational $\left(D_{\mathrm{R}}\right)$ and translational $\left(D_{\mathrm{T}}\right)$ diffusivity, respectively. ${ }^{5,25} k_{\mathrm{B}}$ is the Boltzmann constant, $T$ is temperature, $\eta$ is the viscosity of the solvent, $q$ is the momentum transfer $q=\frac{4 \pi}{\lambda} n \sin \left(\frac{\theta}{2}\right), \theta$ is the scattering angle, $\lambda$ is the wavelength of the scattered waves, and $n$ is the refractive index of the suspension. Equation 10 defines a straight line, and indeed, the results of the statistical analysis of the photon count traces recorded at several scattering angles showed this tendency (Figure $2 \mathrm{~g}$ ). The corresponding line has two independent parameters: a slope and an intercept, which relate to the translational and rotational diffusivities, which in turn are related to the dimensions of the tCNCs. The diffusion coefficients of long and straight rods can generally be expressed by a common form ${ }^{26,27}$

$$
\begin{aligned}
& D_{\mathrm{R}}=\frac{3 k_{\mathrm{B}} T}{\pi \eta} \frac{\ln (L / W)}{L^{3}} \\
& D_{\mathrm{T}}=\frac{k_{\mathrm{B}} T}{3 \pi \eta} \frac{\ln (L / W)}{L}
\end{aligned}
$$

where $L$ and $W$ are the length and width, and $L / W$ is the aspect ratio. Equations 11 and 12 do not take into account whether the ends of the rods are flat or round, which in fact can affect Brownian dynamics when the aspect ratio is small. Given that the tCNCs have a high aspect ratio (Figure 3), this was not a concern in our analysis. The linear regression (Figure $2 \mathrm{~g}$, solid line) determined an intercept of $(40.13 \pm 4.3) \mathrm{s}^{-1}$ and a slope of $(1.45 \pm 0.02) \times 10^{-12} \mathrm{~m}^{-2} \mathrm{~s}^{-1}$. Via eq $10-12$ and by applying the rule of propagation of small independent errors, we obtained an average length $(L)$ of $(1394 \pm 75) \mathrm{nm}$ and width $(W)$ of $(13 \pm 3.4) \mathrm{nm}$. These values are in good agreement with values reported elsewhere, ${ }^{28}$ and are also consistent with our TEM analysis (Figure 3). The TEM micrograph shows that the tCNCs are dominantly straight and exhibit a high aspect ratio. Via image analysis, we estimated a width of $W=24 \pm 7 \mathrm{~nm}$, a length of $L=1858 \pm 620 \mathrm{~nm}$, and an aspect ratio of $L / W=77 \pm 9$. The length and width exhibit a clear correlation with a correlation coefficient $=0.92$.

Silica Particles. TEM micrographs of the $\mathrm{SiO}_{2}$ particles and the results of the image analyses are shown in Figure 4. The orientation-averaged Feret diameters exhibit small dispersions, and the coefficients of variation are below $10 \%$ (Figure $4 a$ ). The mean diameters determined by image analysis are 96, 238, 305, and $397 \mathrm{~nm}$ for $\mathrm{SiO}_{2}-\mathrm{A}, \mathrm{SiO}_{2}-\mathrm{B}, \mathrm{SiO}_{2}-\mathrm{C}$, and $\mathrm{SiO}_{2}-\mathrm{D}$, respectively. As shown by two close-up views, the existence of shape anisotropy-resulting from slight elongation and moderate deformations-is evident. We quantified the degree of shape anisotropy by the circularity (Figure 4b), which measures the deviation of the perimeter of the particle from a perfect sphere with a smooth surface. The circularity was found to be below one-the mean values are $0.92,0.94,0.89$, and 0.92 for $\mathrm{SiO}_{2}-\mathrm{A}, \mathrm{SiO}_{2}-\mathrm{B}, \mathrm{SiO}_{2}-\mathrm{C}$, and $\mathrm{SiO}_{2}-\mathrm{D}$, respectivelyindicating anisotropic polarizability and leading to a small but nonvanishing depolarization ratio. ${ }^{6,29}$

The depolarization ratio of the silica particles is much smaller than that of the tCNCs, and due to their low concentration and relatively large diameter, the intensity of depolarized scattering
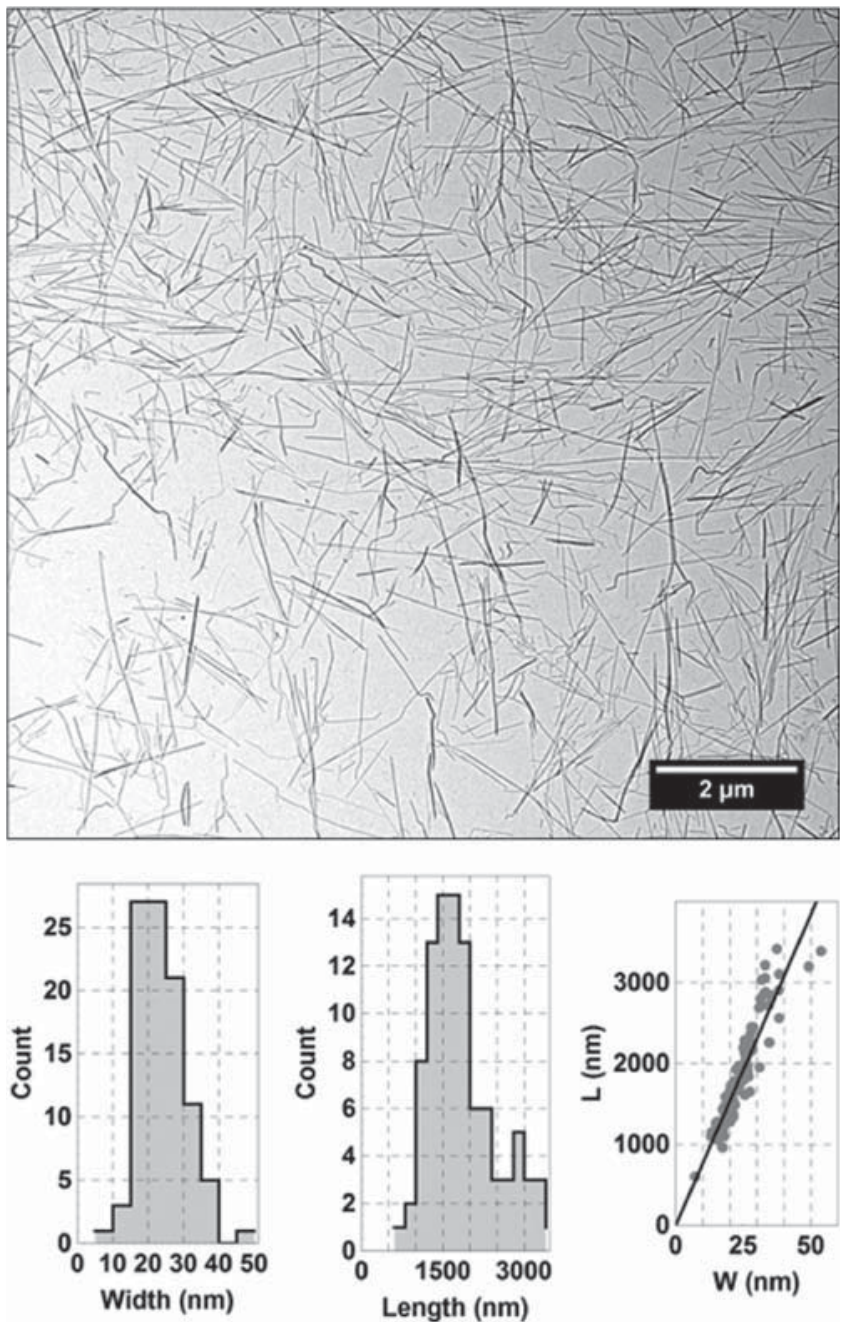

Figure 3. Top: TEM micrograph of the tCNCs used. Bottom: Width and length distribution functions of the tCNCs obtained from TEM image analysis.

in our instrument was only sufficiently high at lower scattering angles. Therefore, we did not access the whole full $q$-range, and restricted our analysis to one angle $\left(\theta=30^{\circ}\right)$. Figure 5 shows the SVS analysis of depolarized light scattered from dilute suspensions of silica particles. The analyses were performed as in the case of tCNCs but this time the intensity autocorrelation function $g_{2}$ was also computed for PCS analysis. The field autocorrelation function $g_{1}$ was obtained from the intensity autocorrelation function, via the Siegert relation $g_{1}=\sqrt{g_{2}-1}$. To determine the relaxation rate via PCS, the experimental spectra were fitted against a single negative exponential function: $g_{1}(t)=\mathrm{e}^{-t / T_{\mathrm{c}}}$ (Figure 5c).

Table 1 lists the correlation times of the fluctuations of the depolarized speckles obtained via SVS and PCS. For comparison, the TEM results are also included. The agreement between the two techniques is indeed very good. The sphereequivalent hydrodynamic radii were estimated via eq 10 , where

$$
D_{\mathrm{R}}=\frac{k_{\mathrm{B}} T}{8 \pi \eta} \frac{1}{r^{3}}
$$

and 

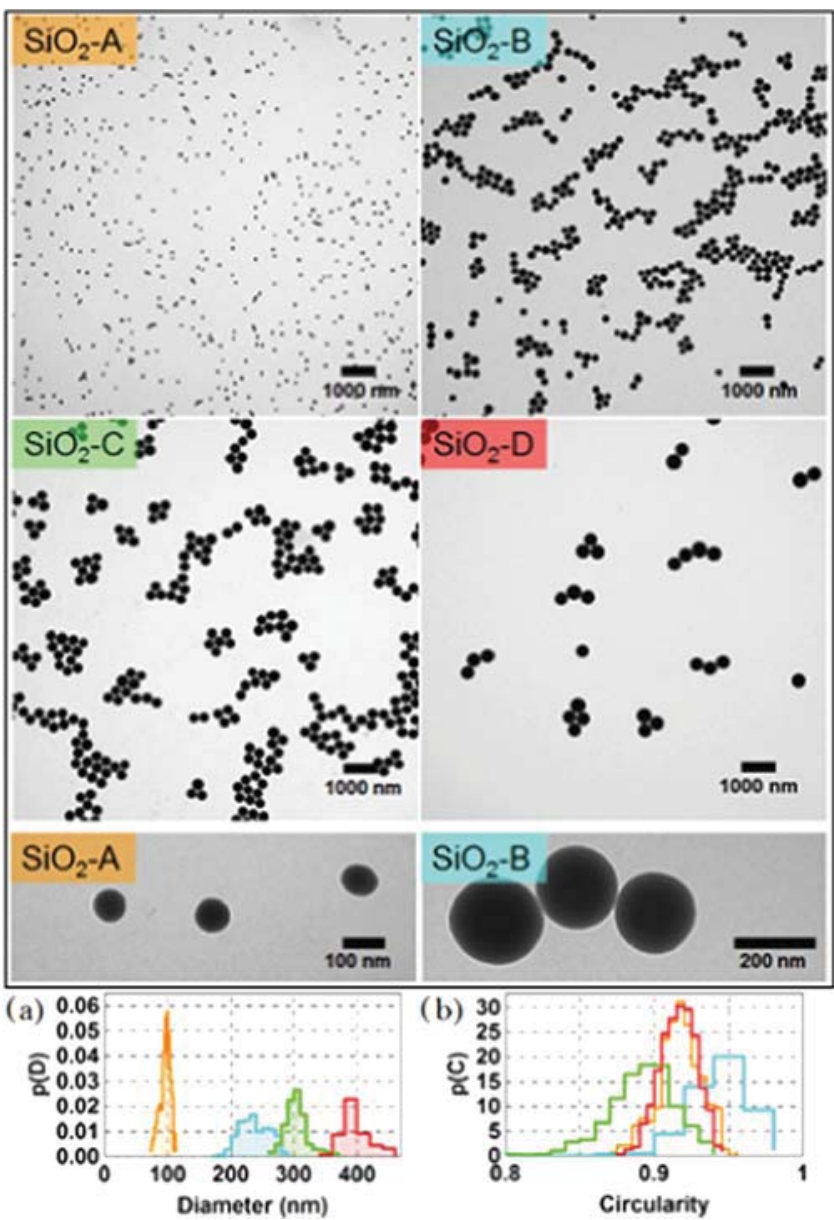

Figure 4. TEM analysis of the $\mathrm{SiO}_{2}$ particles used. In top frame: micrographs and close-up views of $\mathrm{SiO}_{2}$ particles. Bottom: (a) The distributions of the orientation-averaged Feret diameters. Orange: $\mathrm{SiO}_{2}-\mathrm{A}$ (mean $\left.96 \mathrm{~nm}\right)$, cyan: $\mathrm{SiO}_{2}-\mathrm{B}(238 \mathrm{~nm})$, green: $\mathrm{SiO}_{2}-\mathrm{C}(305$ $\mathrm{nm})$, and red: $\mathrm{SiO}_{2}-\mathrm{D}(397 \mathrm{~nm})$. (b) The distributions of the circularities (orange: $\mathrm{SiO}_{2}-\mathrm{A}$, cyan: $\mathrm{SiO}_{2}-\mathrm{B}$, green: $\mathrm{SiO}_{2}-\mathrm{C}$, and red: $\left.\mathrm{SiO}_{2}-\mathrm{D}\right)$.

$$
D_{\mathrm{T}}=\frac{k_{\mathrm{B}} T}{6 \pi \eta} \frac{1}{r}
$$

correspond to the rotational $\left(D_{\mathrm{R}}\right)$ and translational $\left(D_{\mathrm{T}}\right)$ diffusivities of a perfect sphere of radius $r$, respectively. ${ }^{5,25,30-32}$ In comparison to the dimensions established by TEM, both SVS and PCS supplied larger particle sizes, except for $\mathrm{SiO}_{2}-\mathrm{D}$, where the average diameter determined by TEM is slightly larger (6 and $4 \%$, respectively).

\section{DISCUSSION}

We have demonstrated the equivalence of SVS and PCS in determining the coherence times of speckle fluctuations resulting from depolarized light scattering. Our approach has a few potential benefits. First, the theory shows that the statistics of relatively small samples are highly accurate if one has the possibility to collect a sufficiently large number of independent samples. We point out that to not affect the correlation coefficients, PCS requires that the photon trace is fully continuous along its length $\left(t \gg T_{c}\right)$, and that the integration time is much smaller than the relaxation time $(\tau \ll$ $T_{c}$ ). Our approach, on the other hand, is not limited by these requirements and therefore offers more flexibility. In particular, when using one single detector, it enables time-shared multiplexing either between different optical lines aligned to observe different $q$-values and/or between polarized versus depolarized modes (standard DLS vs DDLS), whose combination enables better characterization. ${ }^{33}$ This also waives the necessity of a motorized precision goniometer and the utilization of multiple parallel detection/correlation units, which are required for multiangle DLS apparatus (MALS or MDLS). ${ }^{14,34}$ A lower price-performance ratio is evidently desirable when anticipating an eventual priority of miniaturization and integration of depolarized light scattering into, for example, microfluidic lab-on-a-chip platforms dedicated to automation and parallelization for high-throughput. ${ }^{35-41}$

Moreover, the coherence time can be accurately measured no matter how small $T_{\mathrm{c}}$ is, as long as $\mathrm{Cr} \cdot T_{\mathrm{c}} \gg 1 ; \mathrm{Cr}$ is the counting rate defined as the average number of photons detected per second $\mathrm{Cr}=\langle n(\tau)\rangle \tau^{-1}$. The usual unit of the counting rate is $\mathrm{Hz}$, that is, $\mathrm{Cr}=100 \mathrm{kHz}$ means that on average $10^{5}$ photons are counted in a second. Therefore, a low temporal resolution-even several orders of magnitude smaller than the relaxation rate of the depolarized speckle fluctuation itself-can be sufficiently sensitive for determining Brownian dynamics if one works with high-average photon count rates. This is in striking contrast to photon correlation analysis, which requires a temporal resolution that is several orders of magnitude smaller than the correlation time. This implies that when PCS fails to provide the sufficient temporal resolution and dynamic range, photon counting may solve the problem by replacing the necessity of a high temporal resolution with that of a high counting rate.

In addition, speckle fluctuations represent a stationary and ergodic stochastic process. This, in essence, means that the statistical moments are time invariant and can be estimated from a single but sufficiently large sample of the process, and that time averages and ensemble averages are equivalent. There are noteworthy benefits of exploiting stationarity and ergodicity. Instead of analyzing time averages, one may analyze ensemble averages. For example, by imaging the speckle pattern onto the pixels of a camera, a single "static" snapshot recorded with $\tau$ exposure (as integration time) will, in fact, carry the same information about Brownian dynamics as the temporal photon counts do. Therefore, information describing structure (static light scattering, $\langle n(\tau)\rangle$ ) and Brownian dynamics can be obtained simultaneously at once. Consequently, the statistical analysis on a multitude of single speckles is equivalent to that obtained via one single detector, or equivalently, by one single pixel. Furthermore, a two-dimensional area detector intrinsically provides $q$-space resolved access to Brownian dynamics. Simultaneous access to multiple $q$-values within a single snapshot of $\tau$ exposure is an option that may have considerable advantages, including rapidity and dense information content.

Finally, photon counting offers a means to handle a large intensity burst caused by "foreign" scattering centers (typically dust and aggregates). The presence of outliers and intensity drift deteriorates the correlation function, and hence, corrupts PCS analysis. The negative impact of this can be mitigated only when certain conditions are satisfied. ${ }^{42}$ Although outliers and drift can be easily recognized in a photon stream and rejected once the photon count distribution is constructed, ${ }^{24}$ their realtime exclusion from a photon stream has pitfalls. ${ }^{43}$ 
(a)
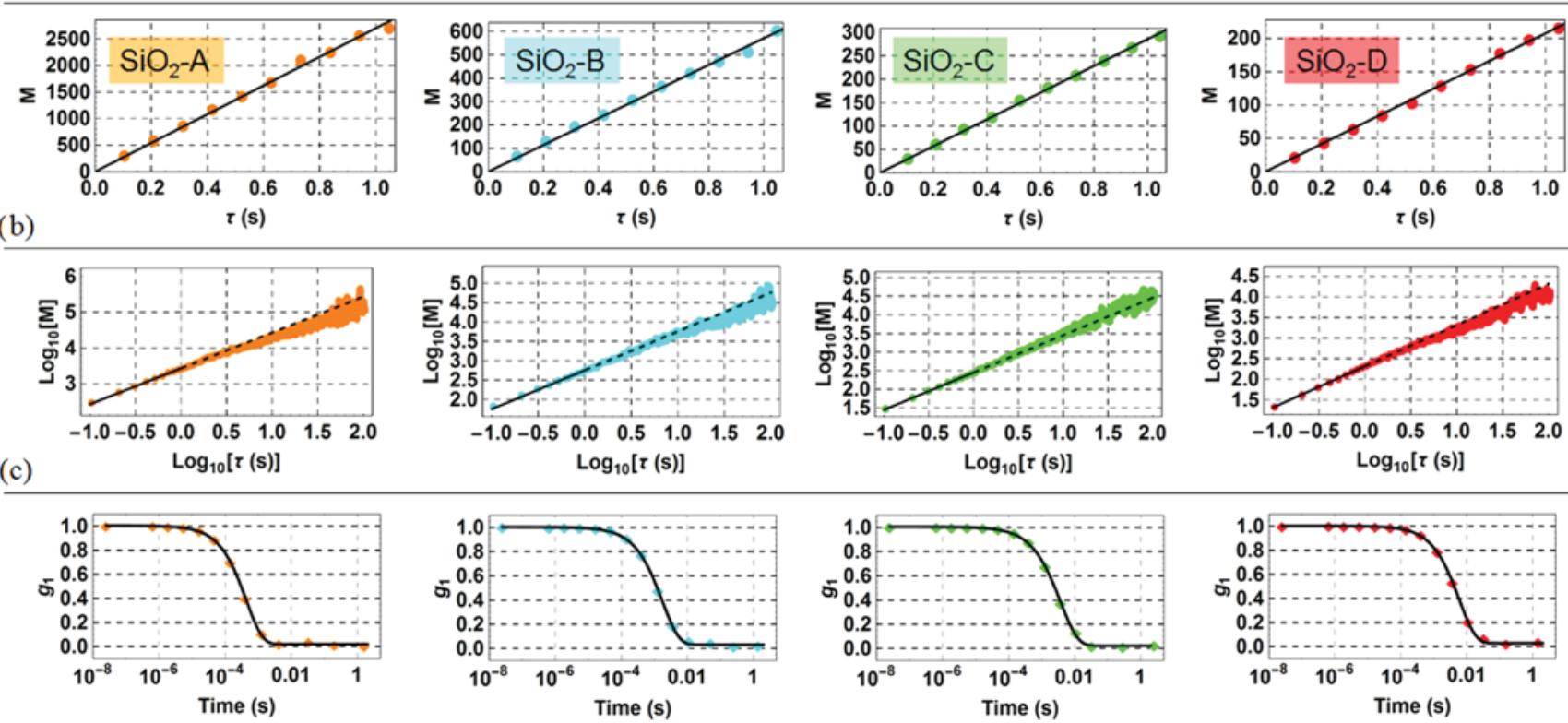

Figure 5. Speckle-visibility spectroscopy (SVS) and PCS analyses of depolarized scattering from the $\mathrm{SiO}_{2}-\mathrm{A}-\mathrm{D}$ particles $\left(\theta=30^{\circ}\right)$. (a) Plots of $M$ as a function of $\tau$ (symbol), and linear regressions (solid lines). (b) Plots of the logarithm of $M$ and $\tau$ on an extended scale $(0.105 \mathrm{~s} \leq \tau \leq 100.5 \mathrm{~s})$. The solid lines are redrawn from panel (a), and the dashed lines are extrapolations of the solid lines. (c) The field autocorrelation functions obtained via PCS and the corresponding fits according to a negative exponential function.

Table 1. Coherence Times and Hydrodynamic Diameters Obtained by SVS and PCS ${ }^{a}$

\begin{tabular}{lcccccc} 
& \multicolumn{2}{c}{ coherence time $T_{\mathrm{c}}(\mu \mathrm{s})$} & & \multicolumn{3}{c}{ diameter $(\mathrm{nm})$} \\
\cline { 2 - 3 } \cline { 6 - 7 } & SVS & PCS & & SVS & PCS & TEM \\
$\mathrm{SiO}_{2}-\mathrm{A}$ & $372 \pm 2$ & $428 \pm 15$ & & $146 \pm 1$ & $153 \pm 2$ & 96 \\
$\mathrm{SiO}_{2}-\mathrm{B}$ & $1809 \pm 19$ & $1706 \pm 72$ & & $255 \pm 1$ & $250 \pm 4$ & 238 \\
$\mathrm{SiO}_{2}-\mathrm{C}$ & $3520 \pm 27$ & $3502 \pm 183$ & & $327 \pm 1$ & $326 \pm 7$ & 305 \\
$\mathrm{SiO}_{2}-\mathrm{D}$ & $5322 \pm 35$ & $5658 \pm 178$ & & $373 \pm 1$ & $382 \pm 5$ & 397
\end{tabular}

${ }^{a}$ Depolarized scattering from $\mathrm{SiO}_{2}-\mathrm{A}-\mathrm{D}$ particles was observed at $\theta=$ $30^{\circ}$. The errors indicated represent standard errors of the parameter fits (SVS, PCS).

\section{SUMMARY}

We presented an experimental approach to study Brownian dynamics through the statistical analysis of depolarized speckles. By identifying a simple linear scaling-which is valid over a broad dynamic range-we established a straightforward relationship between photon count statistics and Brownian dynamics. We treated as well as tested exhaustively the expectable accuracy and precision. Theory and experiments were found to be in very good agreement. To the best of our knowledge, this approach-being analogous to $\mathrm{SVS}^{4}$ and a viable alternative to differential dynamic microscopy ${ }^{44}$-is new to depolarized DLS and can provide benefits. Finally, the theory is also valid for standard polarized scattering if the corresponding relationships are used.

\section{ASSOCIATED CONTENT}

\section{S Supporting Information}

The Supporting Information is available free of charge on the ACS Publications website at DOI: 10.1021/acs.jpcb.7b04971.

Additional analyses and experimental evidence (PDF)

\section{AUTHOR INFORMATION}

\section{Corresponding Author}

*E-mail: sandor.balog@unifr.ch.

\section{ORCID $\odot$}

Christoph Weder: 0000-0001-7183-1790

Alke Petri-Fink: 0000-0003-3952-7849

Sandor Balog: 0000-0002-4847-9845

\section{Author Contributions}

S.B. conceived the project, developed the analytical approach, derived the theoretical treatment, and carried out and analyzed the depolarized light scattering experiments. J.N. isolated the tCNCs and performed their TEM analysis. D.B. and D.A.U. synthesized the $\mathrm{SiO}_{2}$ particles. D.B. collected and edited the TEM micrographs. S.B. wrote the manuscript with contributions of all co-authors. All co-authors have given approval to the final version of the manuscript and none of the authors declares any competing financial interest.

\section{Notes}

The authors declare no competing financial interest.

\section{ACKNOWLEDGMENTS}

The authors are grateful for the financial support of the Adolphe Merkle Foundation and the University of Fribourg. We acknowledge the financial support of the Swiss National Science Foundation through the National Center of Competence in Research Bio-Inspired Materials, the National Research Program "Resource Wood" (136976 and 136911), and Grant \# PP00P2126483/1 (A.P.-F.).

\section{REFERENCES}

(1) Merkus, H. G.; Meesters, G. M. H. Particulate Products: Tailoring Properties for Optimal Performance; Springer International Publishing, 2014.

(2) Borsali, R.; Pecora, R. Soft Matter Characterization; Springer: Netherlands, 2008. 
(3) Goodman, J. W. Statistical Optics; Wiley, 2000.

(4) Bandyopadhyay, R.; Gittings, A. S.; Suh, S. S.; Dixon, P. K.; Durian, D. J. Speckle-Visibility Spectroscopy: A Tool to Study TimeVarying Dynamics. Rev. Sci. Instrum. 2005, 76, No. 093110.

(5) Pecora, R. Dynamic Light Scattering: Applications of Photon Correlation Spectroscopy; Plenum Press: New York, 1985.

(6) Geers, C.; Rodriguez-Lorenzo, L.; Andreas Urban, D.; Kinnear, C.; Petri-Fink, A.; Balog, S. A New Angle on Dynamic Depolarized Light Scattering: Number-Averaged Size Distribution of Nanoparticles in Focus. Nanoscale 2016, 8, 15813-15821.

(7) Levin, A. D.; Shmytkova, E. A.; Khlebtsov, B. N. Multipolarization Dynamic Light Scattering of Nonspherical Nanoparticles in Solution. J. Phys. Chem. C 2017, 121, 3070-3077.

(8) Levin, A. D.; Lobach, A. S.; Shmytkova, E. A. Study of Geometric Parameters of Nonspherical Nanoparticles by Partially Depolarized Dynamic Light Scattering. Nanotechnol. Russ. 2015, 10, 400-407.

(9) Levin, A. D.; Shmytkova, E. A.; Min'kov, K. N. Determination of the Geometric Parameters of Gold Nanorods by Partially Depolarized Dynamic Light Scattering and Absorption Spectrophotometry. Meas. Tech. 2016, 59, 709-714.

(10) Brown, R. G. W.; Smart, A. E. Practical Considerations in Photon Correlation Experiments. Appl. Opt. 1997, 36, 7480-7492.

(11) Kojro, Z.; Riede, A.; Schubert, M.; Grill, W. Systematic and Statistical Errors in Correlation Estimators Obtained from Various Digital Correlators. Rev. Sci. Instrum. 1999, 70, 4487-4496.

(12) Kojro, Z. Influence of Statistical Errors on Size Distributions Obtained from Dynamic Light Scattering Data: Simulation of Experimental Noise in the Intensity Autocorrelation Function. J. Mod. Opt. 1991, 38, 1867-1878.

(13) Schätzel, K. Correlation Techniques in Dynamic Light Scattering. Appl. Phys. B 1987, 42, 193-213.

(14) Bantchev, G. B.; Russo, P. S.; McCarley, R. L.; Hammer, R. P. Simple Multiangle, Multicorrelator Depolarized Dynamic Light Scattering Apparatus. Rev. Sci. Instrum. 2006, 77, No. 043902.

(15) Capadona, J. R.; Van Den Berg, O.; Capadona, L. A.; Schroeter, M.; Rowan, S. J.; Tyler, D. J.; Weder, C. A Versatile Approach for the Processing of Polymer Nanocomposites with Self-Assembled Nanofibre Templates. Nat. Nanotechnol. 2007, 2, 765-769.

(16) Giesche, H. Synthesis of Monodispersed Silica Powders I. Particle Properties and Reaction Kinetics. J. Eur. Ceram. Soc. 1994, 14, 189-204.

(17) Urban, C.; Schurtenberger, P. Characterization of Turbid Colloidal Suspensions Using Light Scattering Techniques Combined with Cross-Correlation Methods. J. Colloid Interface Sci. 1998, 207, 150-158.

(18) Ridler, T. W.; Calvard, S. Picture Thresholding Using an Iterative Selection Method. IEEE Trans. Syst., Man, Cybern. 1978, 8, $630-632$.

(19) Mandel, L.; Sudarshan, E. C. G.; Wolf, E. Theory of Photoelectric Detection of Light Fluctuations. Proc. Phys. Soc. 1964, $84,435$.

(20) Jakeman, E.; Pike, E. R. The Intensity-Fluctuation Distribution of Gaussian Light. J. Phys. A: Gen. Phys. 1968, 1, 128.

(21) McKay, A. T. Distribution of the Coefficient of Variation and the Extended “T” Distribution. J. R. Stat. Soc. 1932, 95, 695-698.

(22) Forkman, J.; Verrill, S. The Distribution of Mckay's Approximation for the Coefficient of Variation. Stat. Probab. Lett. 2008, 78, 10-14.

(23) Peyton, Z.; Peebles, J. Probability, Random Variables, and Random Signal Principles; New York: McGraw-Hill, 1987.

(24) Balog, S.; Zakharov, P.; Scheffold, F.; Skipetrov, S. E. Photocount Statistics in Mesoscopic Optics. Phys. Rev. Lett. 2006, 97, No. 103901.

(25) Debye, P. Polar Molecules; Dover: New York, 1929.

(26) Ortega, A.; Torre, J. G. d. 1. Hydrodynamic Properties of Rodlike and Disklike Particles in Dilute Solution. J. Chem. Phys. 2003, 119, 9914-9919.

(27) Kirkwood, J. G. The General Theory of Irreversible Processes in Solutions of Macromolecules. J. Polym. Sci. 1954, 12, 1-14.
(28) Zhao, Y.; Zhang, Y.; Lindström, M. E.; Li, J. Tunicate Cellulose Nanocrystals: Preparation, Neat Films and Nanocomposite Films with Glucomannans. Carbohydr. Polym. 2015, 117, 286-296.

(29) Khlebtsov, N. G.; Melnikov, A. G.; Bogatyrev, V. A.; Dykman, L. A.; Alekseeva, A. V.; Trachuk, L. A.; Khlebtsov, B. N. Can the Light Scattering Depolarization Ratio of Small Particles Be Greater Than 1/ 3? J. Phys. Chem. B 2005, 109, 13578-13584.

(30) Einstein, A. The Motion of Elements Suspended in Static Liquids as Claimed in the Molecular Kinetic Theory of Heat. Ann. Phys. 1905, 332, 549-560.

(31) Einstein, A. Eine Neue Bestimmung Der Moleküldimensionen. Ann. Phys. 1906, 324, 289-306.

(32) Einstein, A. Berichtigung $\mathrm{Zu}$ Meiner Arbeit: "Eine Neue Bestimmung Der Moleküldimensionen”. Ann. Phys. 1911, 339, 591592

(33) Balog, S.; Rodriguez-Lorenzo, L.; Monnier, C. A.; Michen, B.; Obiols-Rabasa, M.; Casal-Dujat, L.; Rothen-Rutishauser, B.; Petri-Fink, A.; Schurtenberger, P. Dynamic Depolarized Light Scattering of Small Round Plasmonic Nanoparticles: When Imperfection Is Only Perfect. J. Phys. Chem. C 2014, 118, 17968-17974.

(34) Shetty, A. M.; Wilkins, G. M. H.; Nanda, J.; Solomon, M. J. Multiangle Depolarized Dynamic Light Scattering of Short Functionalized Single-Walled Carbon Nanotubes. J. Phys. Chem. C 2009, 113, 7129-7133.

(35) Sorensen, C. M. Light Scattering as a Probe of Nanoparticle Aerosols. Part. Sci. Technol. 2010, 28, 442-457.

(36) Rogers, R. B.; Meyer, W. V.; Zhu, J.; Chaikin, P. M.; Russel, W. B.; Li, M.; Turner, W. B. Compact Laser Light-Scattering Instrument for Microgravity Research. Appl. Opt. 1997, 36, 7493-7500.

(37) Chastek, T. Q.; Beers, K. L.; Amis, E. J. Miniaturized Dynamic Light Scattering Instrumentation for Use in Microfluidic Applications. Rev. Sci. Instrum. 2007, 78, No. 072201.

(38) Destremaut, F.; Salmon, J.-B.; Qi, L.; Chapel, J.-P. Microfluidics with on-Line Dynamic Light Scattering for Size Measurements. Lab Chip 2009, 9, 3289-3296.

(39) Chastek, T. Q.; Iida, K.; Amis, E. J.; Fasolka, M. J.; Beers, K. L. A Microfluidic Platform for Integrated Synthesis and Dynamic Light Scattering Measurement of Block Copolymer Micelles. Lab Chip 2008, $8,950-957$.

(40) Denis, P.; Thomas, Q. C. A Versatile, Low-Cost Approach to Dynamic Light Scattering. Meas. Sci. Technol. 2009, 20, No. 045705.

(41) Chen, Z.; Hu, P.; Meng, Q.; Dong, X. Novel Optical Fiber Dynamic Light Scattering Measurement System for Nanometer Particle Size. Adv. Mater. Sci. Eng. 2013, 2013, 1.

(42) Dynamics of Macromolecular Motion. In Photon Correlation Spectroscopy and Velocimetry; Cummins, H. Z., Pike, E. R., Eds.; Springer, 1977.

(43) Haller, H. R.; Destor, C.; Cannell, D. S. Photometer for Quasielastic and Classical Light Scattering. Rev. Sci. Instrum. 1983, 54, 973-983.

(44) Fabio, G.; Catalina, H.-P.; Roberto, C. Simultaneous Characterization of Rotational and Translational Diffusion of Optically Anisotropic Particles by Optical Microscopy. J. Phys.: Condens. Matter 2016, 28, No. 195201. 